\title{
RELATION BETWEEN RIGHT-TO-LEFT SHUNTS AND SPINAL CORD DECOMPRESSION SICKNESS IN DIVERS
}

\begin{abstract}
The role of right-to-left shunting (RLS) in spinal cord decompression sickness (DCS) remain uncertain and could differ according to the distribution of lesion in spinal cord with a higher risk of upper spinal cord involvement in divers presenting a large patent foramen ovale.

The aims of this study were to assess the prevalence of RLS with transcranial doppler ultrasonography in 49 divers referred for spinal cord DCS and compare it with the prevalence of RLS in 49 diving controls, and to determine a potential relation between RLS and lesion site of spinal cord. The proportion of large RLS was greater in DCS divers than in healthy control divers (odds ratio, 3,6 [95\% CI, 1,3 to 9,5]; $p=0.017$ ). Shunting was not associated with the increased incidence of cervical spinal cord DCS (OR, 1,1 [95\% CI, 0,3 to 3,9]; $p=$ 0.9) while a significant relationship between large RLS and spinal cord DCS with thoracolumbar involvement was demonstrated (OR, 6,9 [95\% CI, 2,3 to 20,4]; $p<0.001$ ). From the above results, we conclude that the risk of spinal cord DCS in divers with hemodynamically relevant RLS is higher than in divers without RLS, particularly in their lower localization.
\end{abstract}

\section{KEY WORDS}

Diving, decompression sickness, spinal cord, transcranial doppler ultrasonography, right-toleft shunting. 


\section{INTRODUCTION}

Divers are at risk of decompression sickness (DCS) resulting from the liberation into blood and tissue of inert gas bubbles previously dissolved within tissues. Usually, gas bubbles infusing to the venous circulation are filtered by the lung, thus preventing entry to the arterial system. The passage of venous gas bubbles either through the pulmonary circulation via a intra-pulmonary (IP) shunt or through an intracardiac patent foramen ovale (PFO) results in arterial gas embolism and the term right-to-left shunting (RLS) is used to describe this incursion of bubbles into the arterial stream.

Numerous studies have shown a high prevalence of PFO in divers who experienced serious neurologic DCS with safe decompression profiles, suggesting that the presence of cardiac RLS was a potential risk factor for the development of DCS $[8,12,19]$. The underlying mechanism may be related with paradoxical venous gas embolism across RLS in situations where the right atrial pressure becomes higher than the left (e.g., release of valsalva maneuver, straining or coughing) [1]. It has been argued that the patency of PFO would contribute to the increased risk of neurologic DCS by 2,5 to $5,5[3,6,14]$. This relative risk (estimated from odds ratio) appeared also higher when the clinical presentation indicated cerebral or cochleovestibular involvement $[5,8]$, associated with short latency of symptoms after surfacing [19] and large PFO [6,8,16].

Spinal cord is the most commonly affected site in neurologic DCS. However, the pathophysiological mechanisms of injury remain to be elucidated even if the prevalent theory focuses more on venous infarction mechanism combined with in situ bubble formation than on arterial bubble embolism mechanism [7].

To date, the relevance of PFO in the pathogenesis of spinal cord DCS is still controversial. While some authors have shown that large PFO appeared to correlate with spinal cord DCS [18], others argued that the presence of PFO was not associated with diving-related spinal cord injury [8]. Actually, it appears that the role of PFO could differ according to the distribution of lesion site in spinal cord, with a higher risk of cervical DCS in PFO divers compared to DCS with thoraco-lumbar involvement [8].

Detection of arterialized micro-bubbles in the middle cerebral artery by transcranial contrast Doppler ultrasonography (TCD) is a noninvasive and reproductible method which has proven to have a high sensitivity and specificity compared with transesophageal echocardiography (TEE) or transthoracic echocardiography (TTE) to assess clinically significant RLS $[10,11]$. From the above, the aims of the present work were: 1) to determine the prevalence of RLS with TCD method in divers referred for spinal cord DCS and compared it with the prevalence in a group of healthy divers and 2) to investigate the relationship between RLS and lesion site (cervical or lower localization).

\section{METHODS}

\section{Subjects}

Forty nine divers (38 men and 11 women; mean age (SD) $46 \pm 12$ years) who suffered from neurologic DCS with symptoms indicative of spinal cord involvement and who were treated in the Ste Anne's military hospital hyperbaric center from 2002 to 2007 entered this study. Diving parameters were as follows: mean maximum depth (SD) $40 \pm 11 \mathrm{msw}$ and mean bottom time (SD) $29 \pm 13 \mathrm{~min}$. None of the divers performed a provocative decompression profile (i.e. fast ascent or omitted decompression stops). Clinical diagnosis of spinal cord injury was made when the criteria of bilateral sensory or/and motor deficit was recognized after the diver surfaced. Cervical disturbance was considered if there was involvement of 
upper limbs while thoraco-lumbar spinal cord injury was retained in case of lower limbs symptoms. If necessary, others characteristic manifestations such as acute back pain or bladder dysfunction were recorded. Combination with patent cerebral or vestibular symptoms was excluded. The mean time from surfacing to initial symptoms was 41 min (range 1-600 $\min$ ).

Forty nine healthy divers (41 men and 8 women; mean age (SD) $41 \pm 7$ years) who had never experienced DCS in the past served as controls. Injured divers and controls were matched for age, gender and diving experience with no significant differences between groups.

All subjects gave their written consent to be examined by TCD ultrasonography for the presence of RLS and ethics committee approval was obtained for the study protocol.

\section{TCD examination}

TCD examinations were performed by experienced investigators according to a strictly standardized method previously described [2]. A 2-MHz pulsed transcranial Doppler device (Pionneer, Nicolet, UK) was used. The patients were lying comfortably on a stretcher. In all patients the right middle cerebral artery was monitored for counting the transient microembolic signals (MES) detected by the probe. The following definition for microembolic signals (MES) was used: typical visible and audible (click, chirp, whistle) short-duration high intensity signal within the Doppler flow spectrum [15]. The investigations were well tolerated by the subjects without side effects.

\section{Contrast Medium}

Microcavitation Contrast Medium (CM) was generated by agitating a mixture of $18 \mathrm{~mL}$ gelofusine and $2 \mathrm{~mL}$ air between two $40 \mathrm{~mL}$ syringes connected by a 3-way stopcock. Once the contrast was prepared, it was injected as a bolus into the antecubital vein in the left arm that had previously been cannulated with a 21 -gauge indwelling intravenous catheter.

\section{Straining maneuver}

The subjects were asked to blow out into a flexible tube with a snorkel mouthpiece connected to a pressure gauge. The timing of the maneuver in the present study follows recent recommendations, taking into account that the contrast agent reaches the right atrium 5 seconds after the injection [13]. The patients were asked to maintain a pressure of $50 \mathrm{mbar}$ for 10 seconds: the patient built up the pressure simultaneously with the start of the CM injection and maintain the pressure at least 5 seconds after the end of the CM injection before releasing the pressure.

Patients were thought to have evidence of a RLS if $>5$ CM-MES were detected in the middle cerebral artery $<20$ seconds after the start of the injection or $<10$ seconds after the release phase. RLS were classified as type I if observed only after the straining maneuver and type II if present at rest with important passage of MES ( $>20)$.

\section{Statistical analysis}

Data are presented as mean $\pm \mathrm{SD}$ or as odds ratio (OR) with $95 \%$ CIs. Differences in RLS prevalence between DCS divers and control divers were calculated by using Chi-square test with Yates's correction or Fisher's exact test where appropriate. Calculation of the OR and 95\% CI was performed by the Woolf method. Statistical tests were run on Sigmastat 3.0 software program (SPSS inc., Chicago, Illinois). The level of significance was set at $\mathrm{p}<0.05$. 


\section{RESULTS}

Of the 49 divers presenting with spinal cord DCS, TCD detected RLS in $25(51 \%)$, whereas control divers demonstrated an RLS in $11(22 \%)$ cases (OR, 3,6 [95\% CI, 1,5 to 8,6]; $\mathrm{p}=$ $0.006)$. Seven of 49 DCS divers (14\%) and 3 of 49 control divers (6\%) had a type I RLS (OR, 3,7 [95\% CI, 0,9 to 15,7]; $\mathrm{p}=0.09$ ) while 18 of 49 DCS divers $(37 \%)$ and 8 of 49 control divers (16\%) had a type II RLS (OR, 3,6 [95\% CI, 1,3 to 9,5]; p =0.017). Table 1

In the subgroup of divers with cervical spinal symptoms of DCS $(n=17)$, the prevalence of RLS was comparable to the prevalence in the control group ( $23 \%$ vs. $22 \%$ respectively; OR, $1,1[95 \% \mathrm{CI}, 0,3$ to 3,9$] ; \mathrm{p}=0.9)$. In contrast, for the subgroup of divers with DCS localizations in the lower spinal cord $(\mathrm{n}=32)$, RLS prevalence was significantly higher than in control divers ( $65 \%$ vs. $22 \%$ respectively, OR, 6,6 [95\% CI, 2,5 to 17,8]; p < 0.001 ). Within this subgroup, the proportion of important shunting (type II) was 50\% compared to the $16 \%$ of the controls (OR, 6,9 [95\% CI, 2,3 to 20,4]; $<<0.001$ ). Table 2

\section{DISCUSSION}

The current study provides evidence that RLS is implicated in spinal cord DCS, specifically in the thoraco-lumbar spinal levels, and it appears that the noted effect on this type of DCS is primarily caused by resting shunt. The prevalence of RLS in the control divers $(22 \%)$ was comparable with those reported in non-diving healthy subjects $(23 \%)$ [10] and in another control group of 101 healthy divers $(24,8 \%)$ [5], excluding a possible selection bias. Our results support the findings of Wilmshurst and coworkers that most divers who presented spinal cord DCS were associated with a large PFO [6]. Nevertheless, evidence to suggest such a link is questioned by others authors. In the Germonpré case-control study, the prevalence of TEE-detected PFO in divers with symptoms indicative of "cerebral" DCS (including high spinal cord and inner ear localization) was significantly higher than the one in matched control divers and in divers having suffered from "spinal" DCS [8]. This has led the author to postulate that large PFO may represent an additional risk factor for occurence of unexplained cerebral, but not low spinal cord, DCS as it may allow the bubbles to bypass the pulmonary circulation, and finally to embolize into the carotid or vertebral arteries. This concept of paradoxical gas embolism through a RLS is also supported by a recent prospective TCD study where the increased incidence of cerebral and cochleovestibular symptoms from DCS was related with major shunts, unlike control divers ( $81 \%$ vs. $24,8 \%$, respectively) [5]. However, in this previous study, a significant association between RLS and spinal cord DCS was equally observed with an OR of 3,9. This estimated risk is comparable with our results, suggesting that the lack of relationship between PFO and spinal cord involvement showed by Germonpré and colleagues should be interpreted with some caution. This discrepancy could be attributed to the uncommon definition of cerebral DCS given by the authors, considering upper spinal cord symptoms as cerebral localization. Furthermore, the small proportion of "spinal" DCS reported in their paper, i.e. 17 cases, might be also a consequence of this discordant statement with our results.

The relevance of RLS in the pathogenesis of spinal cord DCS is still debated. While some authors assumed that arterialization of venous bubbles may be implicated in certain types of spinal cord DCS [5], it has been suggested that dive profile and latency of symptoms could determine whether the spinal cord will be affected. Some dive profiles could result in maximal nitrogen loading of neurological lipid-rich tissues and cause a great liberation of venous bubbles during ascent, so that paradoxical gas embolism through RLS may involve 
bubble amplification in spinal cord and hence promotes DCS after reaching the surface [20].This mechanism could be also consistent with a delay of onset between surfacing and the arterialization of venous bubbles, as demonstrated in animal experiments [7,17], and also confirmed in our study with a mean elapsed time before the occurring of symptoms of $41 \mathrm{~min}$. Actually, the idea of arterial bubbles was already contained in the paper written by Haldane and coworkers in 1908, where they speculated that "if small bubbles are carried through the lung capillaries and pass, for instance, to a slowly desaturating part of the spinal cord, they will there increase in size and may produce serious blockage of the circulation or direct mechanical damage" [4]. Later, the physical aspects of this arterial bubbles scenario were developed by Hennessy, thus introducing the concept of arterial bubble model for decompression tables calculations [9].

In the present work, the risk of developing cervical myelopathy during decompression in divers seemed not related with RLS, implying that mechanism other than paradoxical embolism may be involved in spinal cord DCS. This unexpected finding confirms that this pattern of disease is complex with numerous interactions between the theory of autochthonous bubble formation and the epidural venous infarction hypothesis. Variation in vascular anatomy between the different parts of spinal cord could also explain this discrepancy with the thoraco-lumbar segment. We consider, however, that the clinical features of spinal cord injury classified into 2 distinct entities, i.e. high spinal or low spinal involvement, seems arbitrary and caution should be exercised in interpreting the distribution of spinal cord lesions. Based on our results, we conclude that hemodynamically relevant RLS favours the development of spinal cord DCS in their lower localization. For its relatively ease of execution and excellent sensitivity to detect clinically significant shunts, TCD examination should be performed routinely in neurological DCS assessment. The understanding of the underlying pathophysiologic mechanisms for the presence of RLS in spinal cord DCS remains, however, to be answered. 


\section{References}

1-Balestra C, Germonpre P, Marroni A. Intrathoracic pressure changes after Valsalva strain and other maneuvers: implications for divers with patent foramen ovale. Undersea Hyperb Med 1998; 25: 171-174.

2-Blatteau JE, Peny C, Pontier JM, Gempp E, Louge P. Influence of repetitive open sea dives and physical exercises on right-to-left shunting in healthy divers. Br J Sports Med 2007, doi:10.1136/bjsm.2007.042226 (in press).

3-Bove A. Risk of decompression sickness with patent foramen ovale. Undersea Hyperb Med 1998; 25: 175-178.

4-Boycott AE, Damant C and Haldane JS. The prevention of compressed air illness. J Hyg Cambridge 1908; 8: 342-344

5-Cantais E, Louge P, Suppini A, Palmier B. Right-to-left shunt and risk of decompression illness with cochleo-vestibular and cerebral symptoms in divers: case control study in 101 consecutive dive accidents. Crit Care Med 2003; 31: 84-88

6-Cartoni D, De Castro S, Valente G, Costanzo C, Pellicia A, Beni S. Identification of professional scuba divers with patent foramen ovale at risk for decompression sickness. Am J Cardiol 2004; 94: 270-273.

7-Francis TJR, Mitchell SJ. Pathophysiology of decompression sickness. In: Brubbak AO, Neuman TS (eds). The Bennett and Elliot's physiology and medicine of diving, $5^{\text {th }}$ edn. London:WB Saunders, 2003: 530-556.

8-Germonpré P, Dendale P, Unger P, Balestra C. Patent foramen ovale and decompression sickness in sports divers. J Appl Physiol 1998; 84: 1622-1626.

9-Hennessy TR. On the site of origin, evolution and effects of decompression microbubbles. In: Brubakk AO, Hemmingsen BB, Sundnes G (eds). Supersaturation and bubble formation in fluids and organisms. Trondheim, Norway: Tapir, 1989.

10-Kerut ED, Truax WD, Borreson TE, Van Meter KW, Given MB, Giles TD. Detection of right to left shunts in decompression sickness in divers. Am J Cardiol 1997; 79: 377-378.

11-Klotzsch C, Janssen G, Berlit P. Transesophageal echocardiography and contrast-TCD in the detection of a patent foramen ovale: experiences with 111 patients. Neurology 1994; 44: 1603-1606.

12-Moon RE, Camporesi EM, Kisslo JA. Patent foramen ovale and decompression sickness in divers. Lancet 1989; 1: 513-514.

13-Schwarze J, Sander D, Kukla C, Wittich I, Babikian VL, Klingelhöfer J. Methodological parameters influence the detection of right-to-left shunts by contrast transcranial Doppler ultrasonography. Stroke 1999; 30: 1234-1239. 
14-Schwerzmann M, Seiler C, Lipp E, Guzman R, Lovblad KO, Kraus M, Kucher N. Relation between directly detected patent foramen ovale and ischemic brain lesions in sport divers. Ann Intern Med 2001; 134: 21-24.

15-Spencer M. Detection of cerebral arterial emboli. In: Newell DW, Aaslid R (eds). Transcranial Dopppler. New York: Raven Press Ltd, 1992: 216-230.

16-Torti S, Billinger M, Schwerzmann M, Vogel R, Zbinden R, Windecker S, Seiler C. Risk of decompression illness among 230 divers in relation to the presence and size of patent foramen ovale. Eur Heart J 2004; 25: 1014-1020.

17-Vik A, Jenssen BM, Brubbak AO. Arterial gas bubbles after decompression in pigs with patent foramen ovale. Undersea Biomed Res. 1993; 20: 121-131.

18-Wilmshurst P, Bryson P. Relationship between the clinical features of neurological decompression illness and its causes. Clin Sci 2000; 99: 65-75.

19-Wilmshurst P, Byrne J, Webb-Peploe M. Relation between interatrial shunts and decompression sickness in divers. Lancet 1989; 2: 1302-1306.

20-Wilmshurst P, Davidson C, O'Connell G, Byrne C. Role of cardiorespiratory abnormalities, smoking and dive characteristics in the manifestations of neurological decompression illness. Clin Sci 1994; 84: 297-303. 


\section{Legends}

Table 1

Prevalence of RLS measured by contrast-transcranial Doppler ultrasonography in spinal cord DCS divers and in control divers. Type I RLS corresponds to a contrast passage after valsalva maneuver while type II RLS corresponds to an important contrast passage at rest. Nos in parentheses are $\%$ total. * denotes $p<0.05$ between groups.

Table 2

Prevalence of RLS measured by contrast-transcranial Doppler ultrasonography between DCS divers with cervical involvement and DCS divers with lower spinal cord localization. Nos in parentheses are $\%$ total. $*$ denotes $\mathrm{p}<0.05$ from control divers. 